\title{
Influence of temperature on the transmission performance of track circuit in high-speed railway
}

\author{
Yixuan Yang ${ }^{1,2, *}$, Zhichao Qiao ${ }^{1,2}$, Huiyuan Yin ${ }^{1,2}$, and Jilong $\mathrm{Li}^{1,2}$ \\ ${ }^{1}$ Beijing National Railway Research \& Design Institute of Signal \& Communication Group Co., Ltd. \\ Beijing, China \\ ${ }^{2}$ Beijing Engineering Technology \& Research Center of High-speed Railway Operation Control \\ System, China
}

\begin{abstract}
In order to explore the influence of temperature on track circuit, a mathematical simulation model of track circuit is established. Then, the influence mechanism of temperature on the key equipment of track circuit is analysed. Finally, the influence on the receiver voltage and the locomotive signal current are computed based on the simulation model.
\end{abstract}

\section{Introduction}

Track circuit is an important infrastructure in railway transportation which is still the dominant way to check the train's occupancy in high-speed railway. The relay will be deenergized immediately when a train is occupying the track, and signal indicating prohibition into the block will generate. This makes track circuit the only practical system that can detect trains in real time, as well as a kind of broken rail detection system in the meantime.

The railway network is all over the north and south area of the country, which is extremely cold in the north and very hot in the south. It requires the track circuit equipment to adapt to the temperature range of $-40^{\circ} \mathrm{C} \sim 70^{\circ} \mathrm{C}$. Reference [1] studied the influence of temperature on the acceptance indices of cables by means of testing. The influence of temperature on ferrite permeability and temperature characteristics of special materials was studied in reference [2]. However, the research on the overall performance of temperature track circuit system has not been reported yet. In order to know clearly how the temperature affects the transmission of performance of track circuit signals, a simulation model of the system is established using simulation technology. Then, the influence mechanism of temperature on the key equipment of track circuit is analysed, and the impedance temperature characteristic curve of the device is obtained. Finally, the influence of temperature on the transmission performance of track circuit is analysed based on the simulation model.

\section{Modeling of track circuit}

\footnotetext{
* Corresponding author: yangyixuan@crscd.com.cn
} 


\subsection{Composition of track circuit}

ZPW-2000A track circuit is a unified system of the high-speed railway signal system in China with the utilization ratio of over $99 \%$. The structure and composition of one ZPW2000A track circuit section is shown in figure 1.

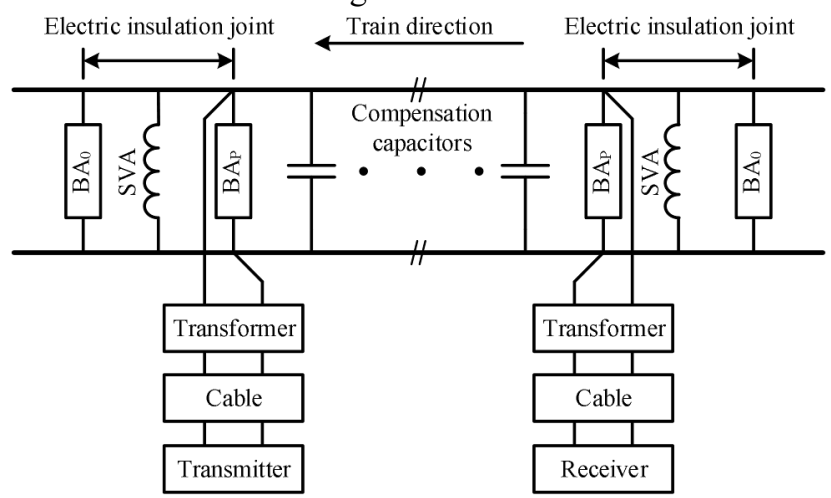

Fig. 1. The structure and composition of typical ZPW-2000A track circuit.

The typical ZPW-2000A track circuit is comprised of indoor devices (transmitter, receiver, etc.), outdoor devices (transformers, SVA, BA0 BAP, etc.), cables connecting them, and the rails. The ZPW-2000A track circuit adopts FSK (frequency shift keying) tuning mode. There are 4 carrier frequencies of the track circuit signal: $1700 \mathrm{~Hz}, 2000 \mathrm{~Hz}$, $2300 \mathrm{~Hz}$ and $2600 \mathrm{~Hz}$. Each section operates at a certain frequency determined by the transmitter.

\subsection{Modeling of the track}

The internal circuits of outdoor devices are quite simple: SVA is an air coil, BA0 and BAP are composed of a few capacitors and inductors. The cable can be modeled according to the transmission line theory. Transmitter and receiver are complex electronic devices, thus the Thevenin equivalent circuits of them are adopted in modeling. Circuits and component parameters are detailed in reference [3].

The keynote is the modeling of track. Usually, the track can be considered as a two-port circuit with ignoring the influence of the earth leakage current as descripted in reference [4] [5]. Under the actual working conditions, the currents of the two rail lines are asymmetrical, which leads to a leakage current into the earth. Therefore, a thorough model is introduced in this paper, where the track circuit is composed of three wires, namely, two rails and the earth line.

The symbolic definitions used in this section are shown in the table 1 .

Table 1. Symbol Table.

\begin{tabular}{|c|l|}
\hline Symbol & \multicolumn{1}{|c|}{ Meaning and Explanation } \\
\hline$z_{1}, z_{2}$ & Self impedance of rail line \\
\hline$g_{1}, g_{2}$ & Ground conductance of rail \\
\hline$g 12$ & The conductance between the ballast surface \\
\hline$z_{\mathrm{M}}$ & Mutual inductance between two rail \\
\hline$x$ & Distance from the beginning of the distance from a rail line \\
\hline$\dot{U}_{1 x}, \dot{U}_{2 x} \dot{I}_{1 x}, \dot{I}_{2 x}$ & The voltage of two rails to the ground, the current in the two rails \\
\hline
\end{tabular}


We assumes that the rail has uniform self-impedance and mutual impedance, and the earth is regarded as a conductor with large cross-sectional area, and the impedance is considered to be zero. The three wires are connected to each other with uniformly distributed leakage conductance. Considering the sinusoidal steady-state solution of a small section of the rail, the equivalent circuit can be drawn as figure 2 .

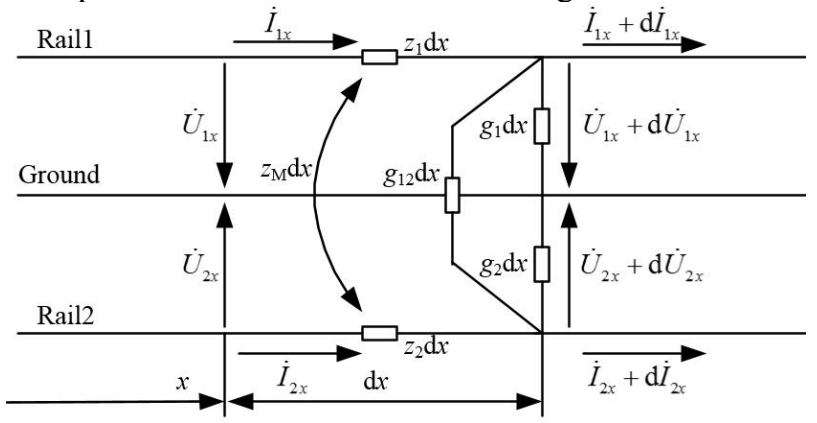

Fig. 2. The equivalent circuit of the rail.

Based on Kirchhoff's law, the circuit equation can be written as:

$$
\left\{\begin{array}{l}
\frac{\mathrm{d} \dot{U}_{1 x}}{\mathrm{~d} x}=-\left(z_{1} \dot{I}_{1 x}+z_{\mathrm{M}} \dot{I}_{2 x}\right) \\
\frac{\mathrm{d} \dot{I}_{1 x}}{\mathrm{~d} x}=-\left(g_{1}+g_{12}\right) \dot{U}_{1 x}+g_{12} \dot{U}_{2 x} \\
\frac{\mathrm{d} \dot{U}_{2 x}}{\mathrm{~d} x}=-\left(z_{2} \dot{I}_{2 x}+z_{\mathrm{M}} \dot{I}_{1 x}\right) \\
\frac{\mathrm{d} \dot{I}_{2 x}}{\mathrm{~d} x}=-\left(g_{2}+g_{12}\right) \dot{U}_{2 x}+g_{12} \dot{U}_{1 x}
\end{array}\right.
$$

which can be simplified as:

$$
\begin{gathered}
\frac{\mathrm{d}^{4} \dot{U}_{1 x}}{\mathrm{~d} x^{4}}-\left[\left(z_{1}\left(g_{1}+g_{12}\right)+z_{2}\left(g_{2}+g_{12}\right)-2 z_{\mathrm{M}} g_{12}\right] \frac{\mathrm{d}^{2} \dot{U}_{1 x}}{\mathrm{~d} x^{2}}\right. \\
+\left(z_{1} z_{2}-z_{\mathrm{M}}^{2}\right)\left(g_{1} g_{2}+g_{1} g_{12}+g_{2} g_{12}\right) \dot{U}_{2 x}=0
\end{gathered}
$$

We can easily get the characteristic equation and the characteristic roots of the upper equation as follows:

$$
\begin{gathered}
\lambda^{4}-a \lambda^{2}+b=0 \\
\lambda_{1,2,3,4}= \pm\left(\frac{1}{2} a \pm\left(\frac{1}{4} a^{2}-b\right)^{1 / 2}\right)^{1 / 2}
\end{gathered}
$$

where $a=\left(z_{1}\left(g_{1}+g_{12}\right)+z_{2}\left(g_{2}+g_{12}\right)-2 z_{\mathrm{M}} g_{12}, b=\left(z_{1} z_{2}-z_{\mathrm{M}}^{2}\right)\left(g_{1} g_{2}+g_{1} g_{12}+g_{2} g_{12}\right)\right.$.

The solution of the equation can be written in the following form:

$$
\left\{\begin{array}{l}
\dot{U}_{1 x}=A_{1} \cosh \lambda_{1} x+A_{2} \sinh \lambda_{1} x+A_{3} \cosh \lambda_{2} x+A_{4} \sinh \lambda_{2} x \\
\dot{U}_{2 x}=M\left(A_{1} \cosh \lambda_{1} x+A_{2} \sinh \lambda_{1} x\right)+N\left(A_{3} \cosh \lambda_{2} x+A_{4} \sinh \lambda_{2} x\right) \\
\dot{I}_{1 x}=y_{11}\left(A_{1} \sinh \lambda_{1} x+A_{2} \cosh \lambda_{1} x\right)+y_{12}\left(A_{3} \sinh \lambda_{1} x+A_{4} \cosh \lambda_{1} x\right) \\
\dot{I}_{2 x}=y_{21}\left(A_{1} \sinh \lambda_{1} x+A_{2} \cosh \lambda_{1} x\right)+y_{22}\left(A_{3} \sinh \lambda_{1} x+A_{4} \cosh \lambda_{1} x\right)
\end{array}\right.
$$


where $\quad C_{1}, \quad C_{2}, \quad C_{3}, \quad C_{4}, \quad A_{1}, \quad A_{2}, \quad A_{3}, \quad A_{4} \quad$ are integral

$$
\begin{aligned}
& \text { constants, } M=\frac{\lambda_{1}^{2}-z_{1}\left(g_{1}+g_{12}\right)+z_{\mathrm{M}} g_{12}}{z_{\mathrm{M}}\left(g_{2}+g_{12}\right)-z_{1} g_{12}}, N=\frac{\lambda_{2}^{2}-z_{1}\left(g_{1}+g_{12}\right)+z_{\mathrm{M}} g_{12}}{z_{\mathrm{M}}\left(g_{2}+g_{12}\right)-z_{1} g_{12}}, \\
& y_{11}=\lambda_{1} \frac{M z_{\mathrm{M}}-z_{2}}{z_{1} z_{2}-z_{\mathrm{M}}^{2}}, y_{12}=\lambda_{2} \frac{N z_{\mathrm{M}}-z_{2}}{z_{1} z_{2}-z_{\mathrm{M}}^{2}}, y_{21}=\lambda_{1} \frac{z_{\mathrm{M}}-M z_{1}}{z_{1} z_{2}-z_{\mathrm{M}}^{2}}, y_{22}=\lambda_{2} \frac{z_{\mathrm{M}}-N z_{1}}{z_{1} z_{2}-z_{\mathrm{M}}^{2}} .
\end{aligned}
$$

Up to now, the formulas obtained above show the variation of voltage and current along the rail line with its primary parameters and the distance $x$ from the transmitter. Furthermore, the equations can be written in a matrix form:

$$
\left[\begin{array}{l}
\dot{U}_{1 x} \\
\dot{U}_{2 x} \\
\dot{I}_{1 x} \\
\dot{I}_{2 x}
\end{array}\right]=\boldsymbol{M}_{\mathrm{UI}}(x)\left[\begin{array}{l}
A_{1} \\
A_{2} \\
A_{3} \\
A_{4}
\end{array}\right]
$$

where $\boldsymbol{M}_{\mathrm{UI}}(x)=\left[\begin{array}{cccc}\cosh \lambda_{1} x & \sinh \lambda_{1} x & \cosh \lambda_{2} x & \sinh \lambda_{2} x \\ M \cosh \lambda_{1} x & M \sinh \lambda_{1} x & N \cosh \lambda_{2} x & N \sinh \lambda_{2} x \\ y_{11} \sinh \lambda_{1} x & y_{11} \cosh \lambda_{1} x & y_{12} \sinh \lambda_{2} x & y_{12} \cosh \lambda_{2} x \\ y_{21} \sinh \lambda_{1} x & y_{21} \cosh \lambda_{1} x & y_{22} \sinh \lambda_{2} x & y_{22} \cosh \lambda_{2} x\end{array}\right]$ is a function of the distance $x$.

Replace the distance function equation with the voltage and current relation equations as follows,

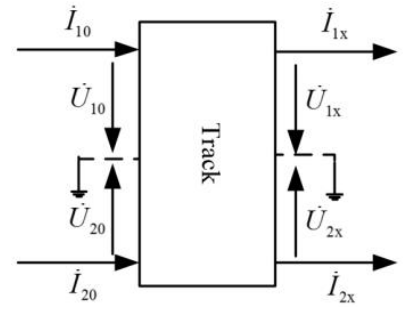

Fig. 3. Equivalent diagram of track circuit.

By substituting it into the formula, the relationship between the distance from the beginning and the voltage and current at the beginning can be obtained as follows:

$$
\left[\begin{array}{c}
\dot{U}_{1 x} \\
\dot{U}_{2 x} \\
\dot{I}_{1 x} \\
\dot{I}_{2 x}
\end{array}\right]=\boldsymbol{M}_{\mathrm{UI}}(x)\left[\begin{array}{c}
A_{1} \\
A_{2} \\
A_{3} \\
A_{4}
\end{array}\right]=\boldsymbol{M}_{\mathrm{UI}}(x) \boldsymbol{M}_{\mathrm{UI}}^{-1}(0)\left[\begin{array}{c}
\dot{U}_{10} \\
\dot{U}_{20} \\
\dot{I}_{10} \\
\dot{I}_{20}
\end{array}\right]
$$

where $\boldsymbol{M}_{\mathrm{UI}}(x) \boldsymbol{M}_{\mathrm{UI}}^{-1}(0)$ can be called the relation matrix of the track circuit.

\subsection{Simulation model of the whole system}

By connecting model of other equipment with the rail model we obtained above, the system model is established, which can be used to analyze the influence of the parameters of any equipment and components on the transmission performance of the track circuit. 


\section{Influence mechanism of temperature}

The track circuit is distributed indoors and outdoors. The indoor temperature is relatively constant, while the outdoor temperature ranges from $-40^{\circ} \mathrm{C}$ to $70^{\circ} \mathrm{C}$. So the most severely affected equipment are the outdoor transmission cable, the electrical insulation device of track side: BAp and BA0.

The main characteristics of track circuit equipment are electrical characteristics, that is, resistance, inductance and capacitance. They are mainly determined by the material characteristics, specifically the resistance corresponding resistivity, the inductance corresponding to the relative permeability, and the capacitance corresponding to the dielectric constant. However, the temperature changes the resistance, inductance and capacitance of the equipment, which is shown in table 2.

Table 2. The relationship between temperature and electrical parameters.

\begin{tabular}{|c|c|c|c|}
\hline Electrical parameters & Material parameters & Relationship with temperature & $\begin{array}{c}\text { Material characteristics in } \\
\text { track circuit }\end{array}$ \\
\hline resistance & resistivity & $\rho=\rho_{0}(1+\alpha t)$ & $\begin{array}{c}\text { Positive temperature } \\
\text { coefficient }\end{array}$ \\
\hline inductance & $\begin{array}{c}\text { Relative } \\
\text { permeability }\end{array}$ & $\mu_{r}=\mu_{r 0}(1+\beta t)$ & $\begin{array}{c}\text { Positive temperature } \\
\text { coefficient }\end{array}$ \\
\hline Capacitance & Permittivity & $\varepsilon=\varepsilon_{0}(1+\gamma t)$ & $\begin{array}{c}\text { Negative temperature } \\
\text { coefficient }\end{array}$ \\
\hline
\end{tabular}

\subsection{Influence mechanism of temperature on the resistance}

Resistance is mainly determined by the resistivity of the material, and the resistivity of the conductor material depends on the nature of the material itself. The resistivity of most materials varies with temperature. In the normal temperature range, the resistivity of metal materials varies linearly with temperature.

The main components that need to be considered are resistors and cables.

(1) Resistance element

The resistance increases with the increase of temperature.

(2) Cables

Cables can be seen as uniform transmission lines. Considering the effect of temperature, the Niemann formula can be written as follows:

$$
R=l\left(\mu_{r} \rho_{0}(1+\alpha t) \omega\right)^{1 / 2} / d
$$

where $\mu_{r}$ is the relative permeability, $\rho_{0}$ is the resistivity, $d$ is the perimeter of the cross section of the conductor, and $l$ is the length of the conductor.

The resistance of the cable varies with temperature in accordance with the above formula, that is, the resistance increases as the temperature increases.

\subsection{Influence mechanism of temperature on the inductance}

Permeability is a physical quantity representing the magnetization of a material. It is the ratio of magnetic induction intensity $\mathrm{B}$ to magnetic field intensity $\mathrm{H}$ in a material. It is also called absolute permeability. The ratio of absolute permeability to vacuum permeability is called relative permeability. The relative permeability range of ferromagnetic material is very wide, for example, the cast iron is $200 \sim 400$, the silicon steel sheet is $7000 \sim 10000$, the Nickel-Zinc ferrite is $10 \sim 1000$, the manganese zinc ferrite is 300 5000. The inductance of 
the component will change when the permeability of the component material changes with temperature.

The main components that need to be considered are inductors, transformers and cables.

(1) Inductors, transformers

The inductance elements in BAp and BA0 are all inductively coupled with iron core. The temperature dependence of relative permeability of $\mathrm{Mn}-\mathrm{Zn}$ ferrites is given in document [2]. It can be seen that the relative permeability increases with the increase of temperature at $-40^{\circ} \mathrm{C} \sim 70^{\circ} \mathrm{C}$.

(2) Cables

The inner core of the cable is copper and the outer shell is aluminum, which are nonferromagnetic materials. The permeability of the cable is relatively small, and the inductance of the cable varies little with temperature.

\subsection{Influence mechanism of temperature on the capacitance}

Dielectric constant is an important data to characterize the electrical properties of dielectric or insulating materials, which is often expressed by $\varepsilon$. It refers to the ratio of capacitance when the same material is used as dielectric and vacuum in the same capacitor, indicating the relative ability of dielectric to store electrostatic energy in electric field. At the same time, dielectric constant is a measure of the capacitive capacity of a capacitor increased by a material relative to a vacuum.

The main components that need to be considered are capacitors and cables.

(1) Capacitors

The capacitors used in BAp and BA0 is CBB capacitors (polypropylene film capacitors). Its temperature coefficient is about minus $300 \mathrm{ppm} /$ degrees, that is, when the temperature rises by $40^{\circ} \mathrm{C}$, the capacitance value decreases by $1 \sim 2 \%$.

(2) Cables

The capacitance of the cable is mainly determined by the medium, which is mainly plastic. Reference [1] indicates that the temperature is positively correlated with the working capacitance.

\subsection{The measured data}

To obtain the real data of the equipment under different temperatures, actual cable, electrical insulation joint equipment (BAp and BA0) are measured in the high and low temperature at $-40 \sim 70^{\circ} \mathrm{C}$. The electric parameters are shown in the table 3 .

Table 3. Measured values of electrical parameters at different temperatures.

\begin{tabular}{|c|c|c|c|c|c|c|c|}
\hline \multirow{2}{*}{ Temperature } & \multicolumn{3}{|c|}{ Cable parameters } & \multicolumn{2}{c|}{ BAp impedance $(\mathrm{m} \Omega)$} & \multicolumn{2}{c|}{ BA0 impedance $(\mathrm{m} \Omega)$} \\
\cline { 2 - 8 } & $\mathrm{R}(\Omega / \mathrm{km})$ & $\mathrm{L}(\mathrm{mH} / \mathrm{km})$ & $\mathrm{C}(\mathrm{nF} / \mathrm{km})$ & real part & imaginary part & real part & imaginary part \\
\hline-40 & 33.2 & 0.803 & 29.23 & 20.52 & -378.26 & 20.42 & -39.52 \\
\hline-20 & 34.1 & 0.804 & 29.21 & 21.39 & -382.34 & 21.32 & -44.94 \\
\hline 0 & 40.5 & 0.803 & 29.17 & 21.32 & -399.58 & 17.76 & -45.25 \\
\hline 30 & 45.4 & 0.867 & 28.02 & 23.38 & -430.67 & 23.53 & -50.37 \\
\hline 50 & 47.2 & 0.821 & 28.92 & 24.42 & -444.59 & 24.10 & -54.25 \\
\hline 70 & 51.1 & 0.814 & 28.88 & 25.73 & -474.41 & 24.71 & -58.77 \\
\hline
\end{tabular}


The measured data shows:

(1) The resistance of cable changes obviously with temperature, namely, the higher temperature, the larger the resistance.

(2) Inductance and capacitance are insensitive to temperature change.

(3) The imaginary parts of BAp tuning equipment (capacitors and inductance elements) are sensitive to temperature and vary greatly.

(4) BA0 tuning equipment is insensitive to temperature and basically unchanged.

\section{Simulation Results}

Besides the measured electrical parameters, the simulation is conducted based on those parameters shown in table 4.

Table 4. Parameters in simulation.

\begin{tabular}{|l|l|l|}
\hline \multicolumn{1}{|c|}{ Parameter } & \multicolumn{1}{c|}{ Value } & \multicolumn{1}{c|}{ Unit } \\
\hline Simulation frequency & 2000 & $\mathrm{~Hz}$ \\
\hline Cable length & 10 & $\mathrm{~km}$ \\
\hline Primary rail circle risistance & 1.177 & $\mathrm{~m} \Omega / \mathrm{m}$ \\
\hline Primary rail circle inductance & 1.314 & $\mu \mathrm{H} / \mathrm{m}$ \\
\hline Rail mutual risistance & 1.678 & $\mathrm{~m} \Omega / \mathrm{m}$ \\
\hline Rail mutual inductance & 0.943 & $\mu \mathrm{H} / \mathrm{m}$ \\
\hline Compensation capacitor & 25 & $\mu \mathrm{F}$ \\
\hline
\end{tabular}

\subsection{Influence on the receiver voltage}

Track circuits of different track length $(100 \mathrm{~m} \sim 1000 \mathrm{~m})$ are simulated to verify the influence of temperature. The voltage curve of the receiving end is shown as below in figure 4. It can be seen that the voltage of the receiving terminal is negatively correlated with the temperature, that is, the terminal voltage gets lower when the temperature increases. It also indicates that the higher of the temperature, the greater transmission loss of the track circuit.

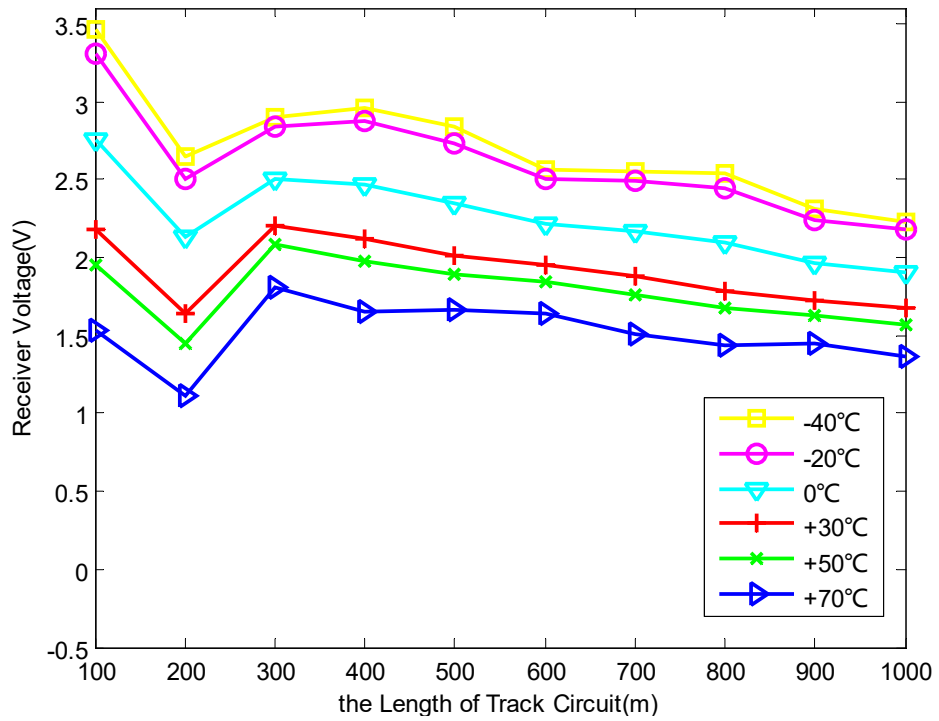

Fig. 4. Receiver voltages under different temperatures. 


\subsection{Influence on the locomotive signal current}

Track circuits of $1000 \mathrm{~m}$ length with different shunt positions are simulated to verify the influence of temperature. The locomotive signal current or short-circuit current is obtained as shown in figure 5. The locomotive signal current shows a trend of periodic oscillation, and the higher the temperature, the smaller the current, which also indicates that the higher the temperature, the greater the transmission loss of track circuit.

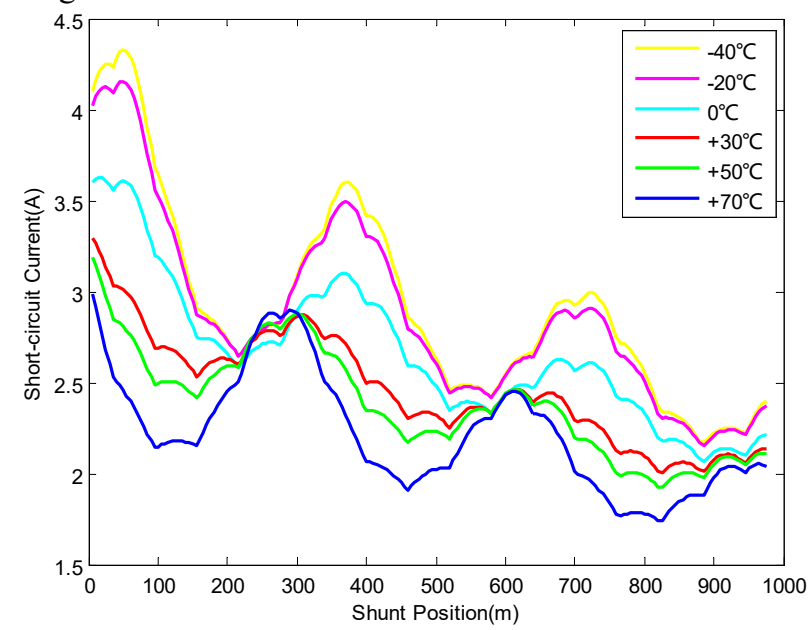

Fig. 5. Locomotive signal current under different temperatures.

\section{Conclusion}

Track circuit is the most basic safety inspection equipment for high-speed railway. Its transmission performance affects the operation efficiency and safety of railway. In this paper, the relationship between the transmission performance of track circuit and temperature is analyzed by combining the simulation model of track circuit and the mechanism of the influence of temperature on electrical parameters, which maybe has a certain guiding significance to the operation of track circuit.

\section{References}

1. Xiayu C 2009. Analysis of factors affecting the characteristics of railway digital signal cable. Railway Signaling \& Communication.

2. Yufeng W 2014. Effects of adding Bi2O3 and glass on the permeability and temperature characteristics of NiCuZn ferrite. Magnetic Materials and Devices.

3. China Railway Corporation 2013. ZPW-2000A jointless FSK automatic block system. Beijing: China Railway Press, pp. 29-105.

4. Nedelchev N 1999. Jointless track circuit length. IEE Proceedings: Electric Power Applications, vol. 146(1), pp.69-74.

5. Mingfu S 1981. Analysis and synthesis of track circuit. Beijing: China Railway Press, pp. $90-94$. 